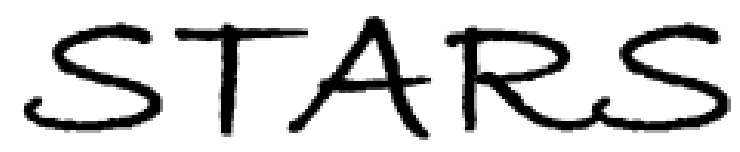

University of Central Florida

STARS

Faculty Bibliography 2010s

Faculty Bibliography

$1-1-2011$

\title{
Local Tomography and the Motion Estimation Problem
}

Alexander Katsevich

University of Central Florida

A. Zamyatin

Find similar works at: https://stars.library.ucf.edu/facultybib2010

University of Central Florida Libraries http://library.ucf.edu

This Article is brought to you for free and open access by the Faculty Bibliography at STARS. It has been accepted for inclusion in Faculty Bibliography 2010 s by an authorized administrator of STARS. For more information, please contactSTARS@ucf.edu.

\section{Recommended Citation}

Katsevich, Alexander and Zamyatin, A., "Local Tomography and the Motion Estimation Problem" (2011).

Faculty Bibliography 2010s. 1465.

https://stars.library.ucf.edu/facultybib2010/1465

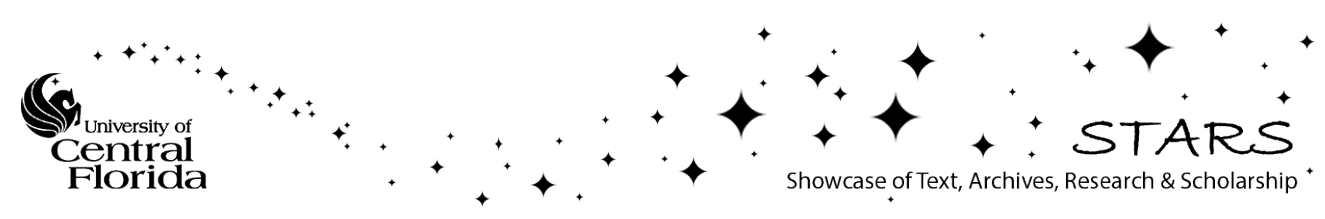




\title{
Local Tomography and the Motion Estimation Problem*
}

\author{
A. Katsevich ${ }^{\dagger}$, M. Silver ${ }^{\ddagger}$, and A. Zamyatin $\ddagger$
}

\begin{abstract}
In this paper we study local tomography (LT) in the motion contaminated case. It is shown that microlocally, away from some critical directions, LT is equivalent to a pseudodifferential operator of order one. LT also produces nonlocal artifacts that are of the same strength as useful singularities. If motion is not accurately known, singularities inside the object $f$ being scanned spread in different directions. A single edge can become a double edge. In such a case the image of $f$ looks cluttered. Based on this observation we propose an algorithm for motion estimation. We propose an empiric measure of image clutter, which we call edge entropy. By minimizing edge entropy we find the motion model. The algorithm is quite flexible and is also used for solving the misalignment correction problem. The results of numerical experiments on motion estimation and misalignment correction are very encouraging.
\end{abstract}

Key words. motion estimation, edge entropy, cone beam

AMS subject classifications. 44A12, 65R10, 92C55

DOI. $10.1137 / 100796728$

1. Introduction. Cardiac (and, more generally, dynamic) imaging is one of the top challenges facing modern computed tomography $(\mathrm{CT})$. When the object being scanned changes during data acquisition the classic tomographic reconstruction theory does not apply. In cardiac CT there are two major groups of approaches for dealing with this issue. One is based on gating, i.e., selecting the CT data which correspond to a fixed cardiac phase, and then using mostly that data for image reconstruction (see, e.g., $[5,9,11,15,19,21]$ ). The second approach, known as motion compensation, is based on incorporating a motion model into a reconstruction algorithm (see, e.g., [3, 6, 10, 14, 24, 31, 32]). Motion compensation algorithms are preferable, because they use all data and have the potential to provide good image quality with reduced x-ray dose. The main difficulty of using such algorithms is that the motion model needs to be known. There are motion estimation algorithms available (see, e.g., [2, 3, 14, 27, 29, 30, 33, 35, 36] for the most recent advances), but significant research still needs to be done to improve efficiency, accuracy, and stability with respect to noise, flexibility, etc.

In this paper we propose a novel approach to motion estimation, which is based on local tomography (LT). See $[1,8,16,22,26,34]$ on cone-beam LT of static objects. The ultimate goal is to come up with a robust algorithm which can reconstruct objects that change during

\footnotetext{
* Received by the editors May 28, 2010; accepted for publication (in revised form) November 3, 2010; published electronically March 9, 2011.

http://www.siam.org/journals/siims/4-1/79672.html

${ }^{\dagger}$ Department of Mathematics, University of Central Florida, Orlando, FL 32816-1364 (akatsevi@mail.ucf.edu). This author's work was supported in part by NSF grant DMS-0806304 and by Toshiba Medical Research Institute USA, Inc.

${ }^{\ddagger}$ Toshiba Medical Research Institute USA, Inc., Vernon Hills, IL 60061 (MSilver@tmriusa.com, AZamyatin@ tmriusa.com).
} 
the scan. Since there is no formula that recovers the object $f$ and motion function $\psi$ from the tomographic data, the most realistic approach to finding $f$ and $\psi$ is via iterations. On the other hand, recovering both of them at the same time would result in an iterative problem of a prohibitively large size. The best approach is to decouple the two tasks, motion estimation and motion compensation, as much as possible. Not all methods achieve this goal. For example, when finding $\psi$ using registration (as is done, e.g., in [14]), one uses the images of $f$ at different times. In other words, finding $\psi$ depends on the knowledge of $f$. This has undesirable consequences. If motion is not known, $f$ is reconstructed with significant artifacts, thereby making subsequent registration unreliable and inaccurate. In contrast, LT is an ideal candidate for decoupling. LT does not reconstruct pointwise values of $f$, but rather a gradient-like image of $f$ with edges enhanced. Thus the only informative feature of LT is the location of edges. We show that if any given edge is seen from the data from two or more source positions, then in the case of incorrectly known motion, the single edge "spreads" and becomes a double edge. As a result, the image looks more cluttered. Our main idea is to iteratively improve the motion model so that image clutter is minimized. We propose an empiric measure of clutter, which we call "edge entropy." Note that the word "entropy" in the name is largely symbolic, since we do not establish any properties that conventional entropy must possess. In the proposed approach, motion estimation is completely independent of our knowledge of $f$, and the desired decoupling is achieved. No knowledge of $f$ is required. The only thing we need is that $f$ possess sufficiently many edges, which is true for practically all $f$ occurring in medical imaging. The use of LT has other benefits as well: (1) LT is very fast. First, it does not require global filtering. Second, backprojection is greatly simplified, since there is no need to compute complicated weights that are mandatory for quasi-exact motion compensating inversion formulas. The weights compensate for variable length of illumination for every voxel in an image. Clearly, high reconstruction speed is critically important for iterative-based motion estimation. (2) LT uses only local data; hence it is not sensitive to data truncation. (3) LT is very flexible and can be used practically with any source trajectory.

Let us mention some other attractive features of the proposed approach. First, it is local in time. Motion estimation is done inside a reasonably short time window, e.g., not much longer than the length of a short scan. This eliminates the need for making the periodicity assumption [4], which frequently holds only approximately. Second, the approach is fairly general and can be used for several types of motion, e.g., cardiac, breathing, etc. Finally, with simple modifications the approach can be applied to solving other practically important problems. As an example we show how to solve a misalignment correction problem for a distorted circular scan. A similar iterative algorithm, which is based on the Feldkamp inversion formula, is proposed in [20]. Since our algorithm is based on LT, it is faster and is not limited to a source trajectory for which accurate and efficient inversion formulas exist. As before, estimation of the unknown source trajectory is completely decoupled from finding $f$, so for the latter purpose one can use any algorithm. For example, when the data are truncated, one might want to use an iterative reconstruction algorithm. If the two problems are coupled, using an iterative algorithm for finding $f$ inside an iterative algorithm for estimating the source trajectory is prohibitively slow.

The paper is organized as follows. In section 2 we propose cone-beam LT function $\mathcal{B} f$ and establish its main properties. In section 3 we study the location and strength of the nonlocal

Copyright (c) by SIAM. Unauthorized reproduction of this article is prohibited. 
artifacts. As opposed to LT in the static case (see [17]), it is not possible here to find the direction of differentiation, which reduces the strength of the artifact by one order in the scale of Sobolev spaces. A similar result for a different geometry was recently reported in [25]. In section 4 we obtain explicit formulas for the shift between the singularities in $\mathcal{B} f$ and $f$ in the case when motion is known incorrectly. A similar result in [17] gives only an implicit relation, and the model used in [17] for describing changes in $f$ is different from the one in this paper. The new motion estimation algorithm as well as a description of the motion model and definition of edge entropy are given in section 6 . Results of numerical experiments on motion estimation and misalignment correction are given in section 7 . Finally, conclusions and directions for future research are stated in section 8 .

2. Local tomography (LT). Let $C$ be a smooth curve in $\mathbb{R}^{3}$ :

$$
I \ni s \rightarrow z(s) \in \mathbb{R}^{3},\left|z_{s}^{\prime}(s)\right| \neq 0,
$$

where $I \subset \mathbb{R}$ is an interval. Usually the source moves along $C$ with constant speed, so we identify $s$ with time variable.

Fix any $s_{0} \in I$. We refer to $s=s_{0}$ as the reference time. To describe the motion inside the object being scanned, we introduce the function $\psi$. Suppose $y=\psi(s, x)$ is the position of the particle at time $s$, which is located at $x$ at the reference time $s=s_{0}$. We assume that for each $s \in I$ the function $\psi(s, x): \mathbb{R}^{3} \rightarrow \mathbb{R}^{3}$ is a diffeomorphism. Physically this means that two distinct points cannot move into the same position. This assumption is quite natural, since cardiac motion is not infinitely compressible (see, e.g., [3]). The inverse of $\psi$ is the function $x=\nu(s, y): \mathbb{R}^{3} \rightarrow \mathbb{R}^{3}$. It gives the original position $x$ of the particle at the reference time, which is located at $y$ at time $s$. We assume that both $\psi$ and $\nu$ are identity maps outside of some open set $U$, which contains the support of the object, and that $\psi, \nu \in C^{\infty}\left(I \times \mathbb{R}^{3}\right)$. As usual, we assume that $C$ is at a positive distance from $U$. Obviously,

$$
\nu(s, \psi(s, x)) \equiv x, \quad \psi(s, \nu(s, x)) \equiv x .
$$

Differentiating the first equation in (2.2) with respect to $s$ and $x$ gives useful identities

$$
\nu_{s}^{\prime}(s, \psi(s, x))+\nabla \nu(s, \psi(s, x)) \psi_{s}^{\prime}(s, x) \equiv 0, \quad \nabla \nu(s, \psi(s, x)) \nabla \psi(s, x) \equiv \mathrm{Id},
$$

where Id is the $3 \times 3$ identity matrix. In (2.3) and everywhere below we use the convention that the operator $\nabla$ acts with respect to space variables. Thus $\nabla \nu(s, y)=\nabla_{y} \nu(s, y)$ and $\nabla \psi(s, x)=\nabla_{x} \psi(s, x)$.

Since matter is conserved, the density at time $s$ and point $y$ is given by $|\nabla \nu(s, y)| f(\nu(s, y))$. Hence the data are

$$
D_{f}(s, \beta):=\int_{0}^{\infty}|\nabla \nu(s, z(s)+t \beta)| f(\nu(s, z(s)+t \beta)) d t, s \in I,
$$

where $\beta$ runs through a subset of the unit sphere determined by the detector. The human tissue is not compressible, so in most cases we can assume $|\nabla \nu(s, y)| \approx 1$ when performing numerical experiments.

Copyright (c) by SIAM. Unauthorized reproduction of this article is prohibited. 
We introduce the following LT function:

$$
(\mathcal{B} f)(x)=\left.\int_{I} \varphi(s, x) \frac{\partial^{2}}{\partial q^{2}} D_{f}(s, \beta(s, x+q \Theta(s, x)))\right|_{q=0} d s,
$$

where

$$
\beta(s, x)=\frac{\psi(s, x)-z(s)}{|\psi(s, x)-z(s)|},
$$

$\Theta(s, x): I \times U \rightarrow \mathbb{R}^{3} \backslash 0$ is a smooth function, and $\varphi \in C_{0}^{\infty}(I \times U)$. Note that (2.5) reduces to equation (2.2) of [17] if $\beta(s, x+q \Theta(s, x))$ is replaced with $\beta(q, x)$. Equation (2.2) of [17] was developed with the goal of reducing as much as possible the global artifact inherent in cone-beam data inversion. The additional flexibility provided by $\Theta$ is needed for increasing computational efficiency (cf. section 7). A slight change in the direction of differentiation away from the optimal one may lead to a significant speed-up at the expense of only a slight increase in the global artifact. The function $\varphi$ in (2.5) determines the time interval, which is used for motion estimation. Define

$$
\Phi(x, s, t):=\nu(s, z(s)+t(\psi(s, x)-z(s))), \quad t>0, s \in I, x \in U .
$$

For a fixed $x \in U$ and $s \in I, \Phi(x, s, t), t>0$, is the preimage of the ray with vertex at $z(s)$ and passing through $\psi(s, x)$. For a fixed $x \in U, \Phi(x, s, t)$ defines a surface parametrized by $s$ and $t$. For convenience, this surface is denoted $\Phi_{x}$. Using (2.3), we get

$$
\begin{aligned}
\Phi_{s}^{\prime}(x, s, t): & =\nu_{s}^{\prime}(s, \check{y})+\nabla \nu(s, \check{y})\left(z_{s}^{\prime}(s)+t\left(\psi_{s}^{\prime}(s, x)-z_{s}^{\prime}(s)\right)\right) \\
& =\nabla \nu(s, \check{y})\left\{\left[z_{s}^{\prime}(s)+t\left(\psi_{s}^{\prime}(s, x)-z_{s}^{\prime}(s)\right)\right]-\psi_{s}^{\prime}(s, \check{x})\right\}, \\
\Phi_{t}^{\prime}(x, s, t): & =\nabla \nu(s, \check{y})(\psi(s, x)-z(s)),
\end{aligned}
$$

where

$$
\check{y}(x, s, t)=z(s)+t(\psi(s, x)-z(s)), \check{x}=\nu(s, \check{y}) .
$$

If $t \neq 1$, then $\check{y}(x, s, t) \neq \psi(s, x)$. The expression in brackets in (2.8) is the velocity of the point $\check{y}$ if we regard it as a fixed point which divides the line segment with endpoints $z(s)$ and $\psi(s, x)$ in the ratio $t: 1-t . \psi_{s}^{\prime}(s, \check{x})$ is the velocity of $\check{y}$ if it moves according to the motion function $\psi$. From (2.8), (2.9), the surface $\Phi_{x}$ is smooth at the point $\Phi(x, s, t)$ if the difference of the two velocities is not parallel to the line segment. We say that $\Phi_{x}$ is smooth if it is smooth at any point $z \in \Phi_{x}, z \neq x$.

Proposition 2.1. Suppose $\Phi_{x}$ is smooth for all $x \in U$. The operator $\mathcal{B}$ defined by $(2.5)$ extends to a map $\mathcal{E}^{\prime}(U) \rightarrow \mathcal{E}^{\prime}(U)$, and

$$
\begin{aligned}
W F(\mathcal{B} f) \subset W F_{v}(f) & \cup E(f, C, \psi), \\
E(f, C, \psi):=\{(x, \eta) & \in T^{*} U \backslash 0:(y, \xi) \in N^{*} \Phi_{x} \cap W F(f), \eta=\xi \cdot \nabla \Phi\left(x, s_{0}, t_{0}\right), \\
y & \left.=\Phi\left(x, s_{0}, t_{0}\right) \neq x,\left(s_{0}, x\right) \in \operatorname{supp} \varphi\right\} .
\end{aligned}
$$

Copyright (C) by SIAM. Unauthorized reproduction of this article is prohibited. 
Here $N^{*} \Phi_{x}$ is the conormal bundle of $\Phi_{x}$. In short, $g$ has an additional singularity at $x$ if $\Phi_{x}$ is tangent to singsupp $f$ at some point $y \neq x$. The singularities of $\mathcal{B} f$, which coincide with those of $f$, are "useful" (from the point of view of practical applications of tomography), while the set $E(f, C, \psi)$ represents the artifact.

Proof. Denote

$$
m:=\inf _{s \in I, x \in U}|x-z(s)|, \quad M:=\sup _{s \in I, x \in U}|x-z(s)|,
$$

and pick $\delta, 0<\delta<m$. Let $w(t)$ be a function with the properties

$$
w(t) \in C_{0}^{\infty}([m-\delta, M+\delta]), w(t)=1, t \in[m, M] .
$$

This function is inserted in the integral (2.4) to ensure that the integration with respect to $t$ is performed over a compact interval, which does not contain $t=0$.

Pick any $g \in C^{\infty}(U)$ and consider the integral

$$
\langle\mathcal{B} f, g\rangle:=\int_{U}(\mathcal{B} f)(x) g(x) d x,
$$

where $f \in C_{0}^{\infty}(U)$. Substituting (2.4) into (2.5) and changing variables according to

$$
t_{1}=t /|\psi(s, x+q \Theta(s, x))-z(s)|,
$$

we get that the argument of $f$ in (2.14) becomes

$$
z=\nu\left(s, z(s)+t_{1}(\psi(s, x+q \Theta(s, x))-z(s))\right) .
$$

Applying (2.2) gives

$$
\nu\left(s, \frac{\psi(s, z)-z(s)}{t_{1}}+z(s)\right)=x+q \Theta(s, x) .
$$

Since $\Theta(s, x)$ is a smooth function, $q$ is restricted to a small neighborhood of zero, and $t_{1}$ is bounded away from zero, it is clear that (2.17) defines a smooth diffeomorphism $z \rightarrow x=$ $X(z, s, t, q)$. Then, (i) taking the derivative with respect to $q$ outside the integral in (2.5), (ii) interchanging the order of integration so that the integral with respect to $x$ in $(2.14)$ becomes the innermost one, and (iii) changing variables $x \rightarrow z$ according to (2.17), we get

$$
\langle\mathcal{B} f, g\rangle=\left\langle f, \mathcal{B}^{*} g\right\rangle,
$$

where $\mathcal{B}^{*} g \in C^{\infty}(U)$. The first assumption of the proposition now follows from continuity.

The proof of (2.11) is given in section 5 below.

Next we compute the principal symbol of $\mathcal{B}$. Besides the smoothness of $\Phi_{x}$, the additional assumptions we make in this calculation are that $(1) \Phi_{s t}^{\prime \prime}(x, s, t=1)$ is never a zero vector and $(2) \Phi_{s t}^{\prime \prime}(x, s, t=1)$ and $\Phi_{t}^{\prime}(x, s, t=1)$ are not parallel. Let us discuss these assumptions. Setting $t=1$ in (2.9) and (2.10) gives $\check{y}=\psi(s, x)$ and

$$
\Phi_{s t}^{\prime \prime}(x, s, t=1)=\frac{d}{d s}[\nabla \nu(s, \psi(s, x))](\psi(s, x)-z(s))+\nabla \nu(s, \psi(s, x))\left(\psi_{s}^{\prime}(s, x)-z_{s}^{\prime}(s)\right) .
$$


From the second equation in (2.3),

$$
\frac{d}{d s}[\nabla \nu(s, \psi(s, x))] \nabla \psi(s, x)+\nabla \nu(s, \psi(s, x)) \nabla \psi_{s}^{\prime}(s, x)=0,
$$

so

$$
\begin{aligned}
& \Phi_{s t}^{\prime \prime}(x, s, t=1) \\
& =\nabla \nu\left(s, \psi(s, x)\left[\left(\psi_{s}^{\prime}(s, x)-z_{s}^{\prime}(s)\right)-\nabla \psi_{s}^{\prime}(s, x) \nabla \nu(s, \psi(s, x))(\psi(s, x)-z(s))\right] .\right.
\end{aligned}
$$

Since $\nu$ is a diffeomorphism, $\Phi_{s t}^{\prime \prime}(x, s, t=1)=0$ is equivalent to

$$
z_{s}^{\prime}(s)=\psi_{s}^{\prime}(s, x)-\nabla \psi_{s}^{\prime}(s, x) \nabla \nu(s, \psi(s, x))(\psi(s, x)-z(s)) .
$$

If the source rotates sufficiently fast compared to the motion of the medium, (2.22) is never satisfied. Comparing (2.21) with (2.8), we get that $\Phi_{s t}^{\prime \prime}(x, s, t=1)$ and $\Phi_{t}^{\prime}(x, s, t=1)$ are not parallel if

$$
\psi(s, x)-z(s) \nVdash\left(\psi_{s}^{\prime}(s, x)-z_{s}^{\prime}(s)\right)-\nabla \psi_{s}^{\prime}(s, x) \nabla \nu(s, \psi(s, x))(\psi(s, x)-z(s)) .
$$

Assuming again that the source rotates sufficiently fast, $(2.23)$ is equivalent to the requirement that the tangent to the source trajectory never points into the region of interest. This is a common condition, which is satisfied by all practical scanning trajectories.

From (2.4) and (2.5) we get

$$
\begin{aligned}
& (\mathcal{B} f)(x)=\int_{\mathbb{R}^{2}} \varphi(s, x) w(t) \\
& \quad \times\left.\frac{\partial^{2}}{\partial q^{2}}|\nabla \nu(s, z(s)+t \beta(s, x+q \Theta))| f(\nu(s, z(s)+t \beta(s, x+q \Theta)))\right|_{q=0} d t d s .
\end{aligned}
$$

Representing $f$ in terms of its Fourier transform and changing variables gives

$$
\begin{aligned}
(\mathcal{B} f)(x) & =\frac{1}{(2 \pi)^{3}} \int_{\mathbb{R}^{3}} \tilde{f}(\xi) B(x, \xi) e^{-i \xi \cdot x} d \xi, \\
B(x, \xi): & =\int_{\mathbb{R}^{2}} Q_{0}(x, \xi, s, t) e^{-i \xi \cdot(\Phi(x, s, t)-x)} d t d s,
\end{aligned}
$$

where $Q_{0}(x, \xi, s, t) \in S^{2}\left(U \times \mathbb{R}^{3}\right)$, the seminorms of $Q_{0}$ as member of the symbol class $S^{2}$ are uniformly bounded with respect to $(s, t) \in \mathbb{R}^{2}$, and the asymptotics of $Q_{0}$ is given by

$$
\begin{aligned}
Q_{0}(x, \xi, s, t)=- & \varphi(s, x) w(t|\psi(s, x)-z(s)|)|\psi(s, x)-z(s)| \\
& \times\left[(t \xi \cdot \nabla \nu(s, \check{y}(x, s, t)) \nabla \psi(s, x) \Theta(s, x))^{2}+O(|\xi|)\right], \quad|\xi| \rightarrow \infty .
\end{aligned}
$$

The term $O(|\xi|)$ in (2.26) is stable when differentiated with respect to $s$ and $t$ any number of times. Using (2.8), (2.9), we get that at the stationary point of the phase

$$
\begin{aligned}
& \xi \cdot \nabla \nu(s, \check{y})\left(\left[z^{\prime}(s)+t\left(\psi_{s}^{\prime}(s, x)-z^{\prime}(s)\right)\right]-\psi_{s}^{\prime}(s, \check{x})\right)=0, \\
& \xi \cdot \nabla \nu(s, \check{y})(\psi(s, x)-z(s))=0 .
\end{aligned}
$$

Copyright (C) by SIAM. Unauthorized reproduction of this article is prohibited. 
If $t=1$, then $x=\check{x}$ and the first equation in (2.27) is trivially satisfied. Hence $(s, t=1)$ is a stationary point if $\xi$ is perpendicular to $\nabla \nu(s, \check{y})(\psi(s, x)-z(s))$, where $\check{y}=\psi(s, x)$. By construction, $\xi \cdot \Phi_{s s}^{\prime \prime}(x, s, t=1) \equiv 0$. Thus the stationary point is nondegenerate if $\xi \cdot \Phi_{s t}^{\prime \prime}(x, s, t=1) \neq 0$. In view of the second equation in $(2.27)$, the critical direction at any $(s, x)$ is given by

$$
\xi_{\mathrm{cr}}(s, x):=\left.\Phi_{t}^{\prime}(x, s, t)\right|_{t=1} \times\left.\Phi_{s t}^{\prime \prime}(x, s, t)\right|_{t=1} .
$$

Let $s_{j}=s_{j}(x, \xi), j=1,2, \ldots$, be the solutions to (2.27) with $t=1$. Assume that $\xi$ is away from a conic neighborhood of the set

$$
\operatorname{Crit}(x):=\left\{\xi \in \mathbb{R}^{3} \backslash 0: \xi=\xi_{\text {cr }}(s, x),(s, x) \in \operatorname{supp} \varphi\right\} .
$$

Then the critical points $\left(s_{j}, t=1\right)$ are nondegenerate, and by the stationary phase method

$$
B(x, \sigma \xi)=-2 \pi \sigma \sum_{j} \varphi\left(s_{j}, x\right)\left|\psi\left(s_{j}, x\right)-y\left(s_{j}\right)\right| \frac{\left|\xi \cdot \Theta\left(s_{j}, x\right)\right|^{2}}{\left|\xi \cdot \Phi_{s t}^{\prime \prime}\left(x, s_{j}, t=1\right)\right|}+O(1), \quad \sigma \rightarrow \infty
$$

Here we have used the second equation in $(2.3)$, the fact that $\Phi(x, s, t=1) \equiv x$, and that the signature of the Hessian of the phase at the stationary point equals zero. If we choose, for example,

$$
\Theta(s, x)=\Phi_{s t}^{\prime \prime}(x, s, t=1)
$$

then $(2.30)$ becomes

$$
B(x, \sigma \xi)=-2 \pi \sigma \sum_{j} \varphi\left(s_{j}, x\right)\left|\psi\left(s_{j}, x\right)-y\left(s_{j}\right)\right|\left|\xi \cdot \Phi_{s t}^{\prime \prime}\left(x, s_{j}, t=1\right)\right|+O(1), \quad \sigma \rightarrow \infty .
$$

3. Artifact. We are now interested in solutions to (2.27) with $t \neq 1$. Similarly to (2.25), we have

$$
(\mathcal{B} f)(x)=\frac{1}{(2 \pi)^{3}} \int \tilde{f}(\xi)\left\{\int Q_{0}(x, \xi, s, t) e^{-i \xi \cdot \Phi(x, s, t)} d s d t\right\} d \xi
$$

Consider the integral with respect to $s$ and $t$. Pick some $x_{0} \in U, s_{0} \in I$, and $t_{0} \neq 1$, and set

$$
\xi_{0}=\Phi_{s}^{\prime}\left(x_{0}, s_{0}, t_{0}\right) \times \Phi_{t}^{\prime}\left(x_{0}, s_{0}, t_{0}\right) .
$$

Suppose that $s=s(x, \xi)$ and $t=t(x, \xi)$ solve the system

$$
\xi \cdot \Phi_{s}^{\prime}(x, s, t)=0, \quad \xi \cdot \Phi_{t}^{\prime}(x, s, t)=0
$$

for $(x, \xi)$ in a conic neighborhood of $\left(x_{0}, \xi_{0}\right)$. In general there can be several solutions, but we are looking for the one close to $\left(s_{0}, t_{0}\right)$. Obviously, $s(x, \xi)$ and $t(x, \xi)$ are homogeneous of degree zero in $\xi$.

Systems (3.3) and (2.27) are the same. However, since $t \neq 1$, no additional insight is gained by representing $\Phi$ in terms of $\psi$ and $\nu$.

Copyright $\odot$ by SIAM. Unauthorized reproduction of this article is prohibited. 
Let $\kappa(x, \xi)$ be the Gaussian curvature of $\Phi_{x}$ at the point $y=\Phi(x, s(x, \xi), t(x, \xi))$. When there is no motion, $\Phi_{x}$ is a ruled surface with zero Gaussian curvature. In the presence of motion we can assume that, generically, $\kappa(x, \xi) \neq 0$. The Hessian of the phase at the stationary point is proportional to the curvature:

$$
\operatorname{det}\left(\begin{array}{ll}
\xi \cdot \Phi_{s s}^{\prime \prime}(x, s, t) & \xi \cdot \Phi_{s t}^{\prime \prime}(x, s, t) \\
\xi \cdot \Phi_{s t}^{\prime \prime}(x, s, t) & \xi \cdot \Phi_{t t}^{\prime \prime}(x, s, t)
\end{array}\right)=\kappa(x, \xi)|\xi|^{2}\left|\Phi_{s}^{\prime}(x, s, t) \times \Phi_{t}^{\prime}(x, s, t)\right|^{2},
$$

where $x, \xi, s$, and $t$ satisfy (3.3). By assumption $\Phi_{s}^{\prime}$ and $\Phi_{t}^{\prime}$ are linearly independent; i.e., $\Phi_{x_{0}}$ is smooth at $y_{0}=\Phi\left(x_{0}, s\left(x_{0}, \xi_{0}\right), t\left(x_{0}, \xi_{0}\right)\right)$. Hence the right-hand side of (3.4) is not zero at $x_{0}, \xi_{0}, s_{0}, t_{0}$, the Hessian is nondegenerate, and the functions $s(x, \xi), t(x, \xi)$ are locally well defined and smooth. By the stationary phase method,

$$
\int Q_{0}(x, \xi, s, t) e^{-i \xi \cdot \Phi(x, s, t)} d s d t=Q_{1}(x, \xi) e^{-i \xi \cdot \Phi(x, s(x, \xi), t(x, \xi))},
$$

where $Q_{1}$ is a symbol from the class $S^{1}$ in a conic neighborhood of $\left(x_{0}, \xi_{0}\right)$ (cf. (2.26)), and the asymptotics of $Q_{1}$ is given by

$$
Q_{1}(x, \xi)=c \frac{Q_{0}(x, \xi, s(x, \xi), t(x, \xi))}{\left|\operatorname{det}\left(\xi \cdot \Phi^{\prime \prime}\right)\right|^{1 / 2}}+O(1), \quad|\xi| \rightarrow \infty
$$

where $\xi \cdot \Phi^{\prime \prime}$ is the matrix in (3.4) and constant $c$ incorporates some powers of $2 \pi$ and $i$. Combine (3.1) and (3.6):

$$
(\mathcal{B} f)(x)=\frac{1}{(2 \pi)^{3}} \int \tilde{f}(\xi) Q_{1}(x, \xi) e^{-i a(x, \xi)} d \xi,
$$

where $a(x, \xi):=\xi \cdot \Phi(x, s(x, \xi), t(x, \xi))$. If $\operatorname{det}\left(\partial^{2} a(x, \xi) / \partial x \partial \xi\right) \neq 0$ at $\left(x_{0}, \xi_{0}\right)$, then locally (3.7) is a Fourier integral operator (FIO) associated with a canonical transformation, and the order of the operator equals one (see [13, pp. 25-26]). In view of (2.30) this means that the artifacts and useful singularities can be of the same strength in the scale of Sobolev spaces.

4. Incorrectly known motion. Suppose that instead of the motion function $\psi$ we know its approximation $\psi_{\epsilon}(s, x)=\psi(s, x)+\epsilon \psi_{1}(s, x)$. In this case the function $\Phi$ of $(2.7)$ is replaced by

$$
\Phi(\epsilon, x, s, t):=\nu\left(s, z(s)+t\left(\psi_{\epsilon}(s, x)-z(s)\right)\right) .
$$

Consequently, the useful singularities of $\mathcal{B} f$ no longer coincide with the singularities of $f$. To find the shift between them we assume that the error in $\psi_{\epsilon}$ is small, i.e., $\epsilon \rightarrow 0$, and find the first order approximation of the shift. If $\epsilon=0$, the function $s(x, \xi)$ is determined from (3.3) with $t(x, \xi) \equiv 1$. If $\epsilon \neq 0$, we have to solve

$$
\xi \cdot \Phi_{s}^{\prime}\left(\epsilon, x_{\epsilon}, s_{\epsilon}, t_{\epsilon}\right)=0, \quad \xi \cdot \Phi_{t}^{\prime}\left(\epsilon, x_{\epsilon}, s_{\epsilon}, t_{\epsilon}\right)=0, \quad \Phi\left(\epsilon, x_{\epsilon}, s_{\epsilon}, t_{\epsilon}\right)=\Phi(0, x, s, t=1) .
$$

Equations (4.2) mean that the singularity of $f$ at $x$ is mapped into the singularity of $\mathcal{B} f$ at $x_{\epsilon}$. Set $x_{\epsilon}=x+\Delta x, s_{\epsilon}=s+\Delta s, t_{\epsilon}=1+\Delta t$. Expanding (4.2) in the Taylor series around 
$\epsilon=0$, using (3.3), and keeping the first order terms in $\epsilon$ gives

$$
\begin{aligned}
& \epsilon \xi \cdot \Phi_{\epsilon s}^{\prime \prime}+\xi \cdot \nabla \Phi_{s}^{\prime} \Delta x+\xi \cdot \Phi_{s s}^{\prime \prime} \Delta s+\xi \cdot \Phi_{s t}^{\prime \prime} \Delta t=0, \\
& \epsilon \xi \cdot \Phi_{\epsilon t}^{\prime \prime}+\xi \cdot \nabla \Phi_{t}^{\prime} \Delta x+\xi \cdot \Phi_{s t}^{\prime \prime} \Delta s+\xi \cdot \Phi_{t t}^{\prime \prime} \Delta t=0, \\
& \epsilon \Phi_{\epsilon}^{\prime}+\nabla \Phi \Delta x+\Phi_{s}^{\prime} \Delta s+\Phi_{t}^{\prime} \Delta t=0 .
\end{aligned}
$$

All the derivatives in (4.3) are computed at $\epsilon=0, x, s=s(x, \xi)$, and $t=1$. Since $\Phi(0, x, s, t=$ $1) \equiv x$, it follows that $\nabla \Phi_{s}^{\prime}=0, \Phi_{s s}^{\prime \prime}=0, \Phi_{s}^{\prime}=0$. The first and third equations in (4.3) yield

$$
\Delta t=-\epsilon \frac{\xi \cdot \Phi_{\epsilon s}^{\prime \prime}}{\xi \cdot \Phi_{s t}^{\prime \prime}}, \quad \Delta x=\epsilon(\nabla \Phi)^{-1}\left[\Phi_{t}^{\prime} \frac{\xi \cdot \Phi_{\epsilon s}^{\prime \prime}}{\xi \cdot \Phi_{s t}^{\prime \prime}}-\Phi_{\epsilon}^{\prime}\right] .
$$

When $t=1, \nabla \Phi=\mathrm{Id}$ and

$$
\Phi_{t}^{\prime}=\nabla \nu(\psi(s, x)-z(s)), \quad \Phi_{\epsilon}^{\prime}=\nabla \nu \psi_{1}(s, x) .
$$

Substituting into (4.4) gives

$$
\Delta x=\epsilon \nabla \nu(s, \psi(s, x))\left[c(\psi(s, x)-z(s))-\psi_{1}(s, x)\right]+O\left(\epsilon^{2}\right), \quad c=\frac{\xi \cdot \Phi_{\epsilon s}^{\prime \prime}}{\xi \cdot \Phi_{s t}^{\prime \prime}} .
$$

By our assumption $\xi \cdot \Phi_{s t}^{\prime \prime} \neq 0$, so $\Delta x$ is indeed of order $O(\epsilon), \epsilon \rightarrow 0$.

5. End of proof of Proposition 2.1. Using a partition of unity we may suppose that $W F(f)$ is a subset of a sufficiently small conic neighborhood of $\left(x_{0}, \xi_{0}\right) \in T^{*} U$ and that $Q_{0} \equiv 0$ (cf. (2.26) and (3.1)) for $(s, t)$ outside a sufficiently small neighborhood of $\left(s_{0}, t_{0}\right) \in I \times \mathbb{R}_{+}$. Initially we consider the case $t_{0} \neq 1$. First of all, from $(2.4)$ and $(2.5),(\mathcal{B} f)(x) \equiv 0$ outside a neighborhood of $y_{0}=\Phi\left(x_{0}, s_{0}, t_{0}\right)$. In view of the partition of unity, (2.4) needs to be modified by including a cut-off function depending on $t$. Passing to a finer partition of unity if necessary, $(3.1)$ implies that $(\mathcal{B} f)(x)$ is smooth near $y_{0}$ unless $\xi_{0}$ is parallel to $\Phi_{s}^{\prime}\left(x_{0}, s_{0}, t_{0}\right) \times \Phi_{t}^{\prime}\left(x_{0}, s_{0}, t_{0}\right)$. If the two vectors are parallel, we multiply (3.1) by $\varphi_{1}(x) e^{i x \cdot \eta_{0}}$, where $\varphi_{1} \in C_{0}^{\infty}$ and is supported in a neighborhood of $y_{0}$, and $\eta_{0} \neq \xi_{0} \cdot \nabla \Phi\left(x_{0}, s_{0}, t_{0}\right)$. Integrating with respect to $x$ and using the standard argument (see, e.g., [7, p. 114]), we get that $\left(y_{0}, \eta_{0}\right) \notin W F(\mathcal{B} f)$.

Suppose now that $t_{0}=1$. Since $\Phi(x, s, t=1) \equiv x$ and $\xi_{0}=\xi_{0} \cdot \nabla \Phi\left(x_{0}, s_{0}, t_{0}=1\right)$, we get as before that $\left(x_{0}, \xi_{0}\right) \notin W F(f)$ implies $\left(x_{0}, \xi_{0}\right) \notin W F(\mathcal{B} f)$, and (2.11) is proved.

6. A motion estimation algorithm. The proposed algorithm is based on LT. In the case of static objects, the discontinuities (or edges) of $f$ and $\mathcal{B} f$ generally coincide $[8,12,17,22]$ (except for the added singularities or artifacts in $\mathcal{B} f$ and the singularities of $f$ that are invisible from the data). As mentioned in section 4, if there is some uncompensated motion in $f$, the edges of $f$ and $\mathcal{B} f$ no longer coincide. Practically this means that if motion is not known (or is known incorrectly), edges in the reconstructed image spread out. A single edge produces multiple edges at several locations. Consequently, the reconstructed image looks cluttered or random. We can use a measure of randomness in the reconstructed image $\mathcal{B} f$ to gauge whether our motion model is accurate or not. In what follows we call this measure "edge entropy." Using this idea, we summarize the proposed motion estimation algorithm as follows:

1. Assume some motion model. 
2. Perform motion-compensated LT image reconstruction using current motion model.

3. Compute edge entropy of the LT image.

4. If edge entropy is low (i.e., the edges have not spread too much), stop. If edge entropy is high, change the motion model and go to step 2.

A similar idea was used in [20] for misalignment correction in circular cone-beam CT. The main novelty of our approach is that we use LT instead of global Feldkamp-type reconstruction. On the one hand, the use of LT allows us to significantly speed up the iterations. On the other hand, many tools that work with conventional images (most notably, image entropy) do not work with LT images, so we had to develop alternative tools from scratch. We now describe the key steps of the algorithm in more detail.

6.1. Motion model. Let $\left[s_{l}, s_{r}\right] \subset I$ be a parameter/time window, which is used for motion estimation. The center point $s_{0}=\left(s_{l}+s_{r}\right) / 2$ is taken as reference time. The primary purpose of the algorithm is to perform local (in time) motion estimation; thus the width of the window $S:=s_{r}-s_{l}$ is usually rather short. In our experiments $S$ is typically less than one gantry rotation. Let $D \subset U$ denote the region where motion takes place. We assume that $D$ is a rectangle; i.e., $D:=\left\{\left(x_{1}, x_{2}, x_{3}\right) \in \mathbb{R}^{3}: L_{k} \leq x_{k} \leq R_{k}, k=1,2,3\right\}$. To represent motion, we consider a regular grid over $D$. The grid planes are

$$
x_{k}=\zeta_{i k}:=L_{k}+i \Delta x_{k}, \quad 0 \leq i \leq N_{k}+1, k=1,2,3
$$

where $\Delta x_{k}=\left(R_{k}-L_{k}\right) /\left(N_{k}+1\right)$ is the step-size along the $k$ th axis. Thus, grid (6.1) has $N_{1} N_{2} N_{3}$ interior nodes, and for each direction $k$ there are $N_{k}+2$ planes $x_{k}=\zeta_{0 k}, \ldots, x_{k}=$ $\zeta_{N_{k}+1, k}$. Because of motion, the grid planes deform over time:

$$
\begin{array}{ll}
x_{1}=\zeta_{i 1}+a_{i 1}(s) \phi\left[\left(x_{2}-L_{2}\right) /\left(R_{2}-L_{2}\right)\right] \phi\left[\left(x_{3}-L_{3}\right) /\left(R_{3}-L_{3}\right)\right], & 1 \leq i \leq N_{1}, \\
x_{2}=\zeta_{i 2}+a_{i 2}(s) \phi\left[\left(x_{1}-L_{1}\right) /\left(R_{1}-L_{1}\right)\right] \phi\left[\left(x_{3}-L_{3}\right) /\left(R_{3}-L_{3}\right)\right], & 1 \leq i \leq N_{2}, \\
x_{3}=\zeta_{i 3}+a_{i 3}(s) \phi\left[\left(x_{1}-L_{1}\right) /\left(R_{1}-L_{1}\right)\right] \phi\left[\left(x_{2}-L_{2}\right) /\left(R_{2}-L_{2}\right)\right], & 1 \leq i \leq N_{3} .
\end{array}
$$

Each line in (6.2) defines a separate surface, which corresponds to a deformation of one of the original planes (6.1). We assume that motion equals zero at the boundary of $D$, so the boundary grid planes (i.e., those given by $x_{k}=\zeta_{i k}, i=0$, or $N_{k}+1, k=1,2,3$ ) do not deform. In (6.2), the function $\phi$ is smooth, defined on the interval $[0,1]$, and equals zero at both endpoints of the interval. Since the time window $\left[s_{l}, s_{r}\right]$ is sufficiently short, we assume that the functions $a_{i k}(s)$ are linear:

$$
a_{i k}(s)=a_{i k}\left(s-s_{0}\right) /(0.5 S), \quad k=1,2,3,
$$

where $a_{i k}, 1 \leq i \leq N_{k}, k=1,2,3$, are constants to be determined. Note that substituting $s=s_{0}$ into (6.2) gives the rectangular grid (6.1). Equations (6.2) and (6.3) allow us to describe motion of every point in $D$. To determine where a node from the original grid (6.1) is located at time $s$, we identify the three planes where the node is located, deform them according to (6.2), and then find the point of intersection of the three resulting surfaces. Location of all other pixels is computed using trilinear interpolation.

Copyright (C) by SIAM. Unauthorized reproduction of this article is prohibited. 
6.2. Edge entropy. Suppose $\mathcal{B} f$ is computed on a regular grid $\left(x_{i_{1}}, x_{i_{2}}, x_{i_{3}}\right), 1 \leq i_{k} \leq M_{k}$, $k=1,2,3$, which covers $D$. Suppose, for simplicity, that the step-size of the grid is the same along every axis and equals $\Delta x$. Nodes of the grid are denoted $x_{I}:=\left(x_{i_{1}}, x_{i_{2}}, x_{i_{3}}\right)$, where $I=\left(i_{1}, i_{2}, i_{3}\right)$. Of course, this grid should be much more dense than the one in (6.1) $\left(M_{k} \gg\right.$ $\left.N_{k}\right)$. We also need a shifted grid with nodes $\bar{x}_{I}:=\left(\bar{x}_{i_{1}}, \bar{x}_{i_{2}}, \bar{x}_{i_{3}}\right)$, where $\bar{x}_{i_{k}}=x_{i_{k}}+\Delta x / 2$, $1 \leq i_{k} \leq M_{k}-1, k=1,2,3$. We introduce the distance function:

$$
\operatorname{dist}\left(\bar{x}_{I}, \bar{x}_{J}\right)=\max \left(\left|i_{1}-j_{1}\right|,\left|i_{2}-j_{2}\right|,\left|i_{3}-j_{3}\right|\right) .
$$

Calculation of edge entropy consists of several steps. Let parameter $\kappa, 0<\kappa<1$, be fixed.

1. Using finite differences, compute the norm of the gradient at the nodes of the shifted grid $\left|\nabla(\mathcal{B} f)\left(\bar{x}_{I}\right)\right|$.

2. Compute the empirical histogram of the norm of the gradient.

3. Using the histogram, estimate the value $M$ such that $\left|\nabla(\mathcal{B} f)\left(\bar{x}_{I}\right)\right|>M$ for $100 \kappa \%$ of the points (such points are called "bright").

4. By running a sliding window over the image, compute the total number of points $\bar{x}_{I}$ whose distance (in the sense of (6.4)) to the closest bright point equals either 2, 3, or 4 .

5. Divide this number by the total number of nodes in the grid and multiply by 100 (to get percents). The result is the edge entropy of the image $\mathcal{B} f$.

7. Numerical experiments. The original phantom is a superposition of seven balls as shown in Figures 1-3. The motion of the medium is described by the function

$$
\begin{aligned}
& \psi(s, x)=x+ \begin{cases}25 \cos \left(0.35\left(s-s_{0}\right)\right) \Theta, & |x|<10 \\
25 \cos \left(0.35\left(s-s_{0}\right)\right) \frac{75-|x|}{65} \Theta, & 10 \leq|x|<75 \\
0, & |x| \geq 75\end{cases} \\
& \Theta=\left(\cos \theta_{2} \cos \theta_{1}, \cos \theta_{2} \sin \theta_{1}, \sin \theta_{2}\right) .
\end{aligned}
$$

Here $s_{0}$ is reference time, $\theta_{1}=70^{\circ}, \theta_{2}=30^{\circ}$. In this section the units of length are always $\mathrm{mm}$. The detector array is curved and passes through the isocenter. Pixel size on the detector is 0.5 along columns and $10^{-3}$ radians along rows. The source trajectory is circular: $x_{1}=$ $R \cos s, x_{2}=R \sin s, x_{3}=0$, and the source-to-isocenter distance is $R=600$. There are 1000 projections per one rotation, $0 \leq s<2 \pi$. The time corresponding to projection 501 was chosen as reference time: $s_{0}=\pi$. For motion estimation we used only the data corresponding to the range of projections $[101,900]$. The data are simulated using ray tracing as described, e.g., in [18]. Following the common practice in medical imaging, we did not track the changes in density due to motion (see, e.g., [3, 28]). This is equivalent to setting $|\nabla \nu|=1$ in (2.4).

The function $\mathcal{B} f$ is computed on the $112 \times 112 \times 112$ regular grid covering the cube $-75 \leq x_{k} \leq 75, k=1,2,3$. To make the resulting algorithm as numerically efficient as possible, we use the simplest version of the motion-compensated LT. To this end the derivative $\partial^{2} / \partial q^{2}$ in (2.5) is replaced by the second derivative of the cone-beam data along detector rows.

Let $D_{l}$ be a box-like region bounded by six neighboring planes (6.1). As is easily seen, the values of $\mathcal{B} f(x)$ for all $x \in D_{l}$ depend only on the six parameters describing the deformation

Copyright $\odot$ by SIAM. Unauthorized reproduction of this article is prohibited. 


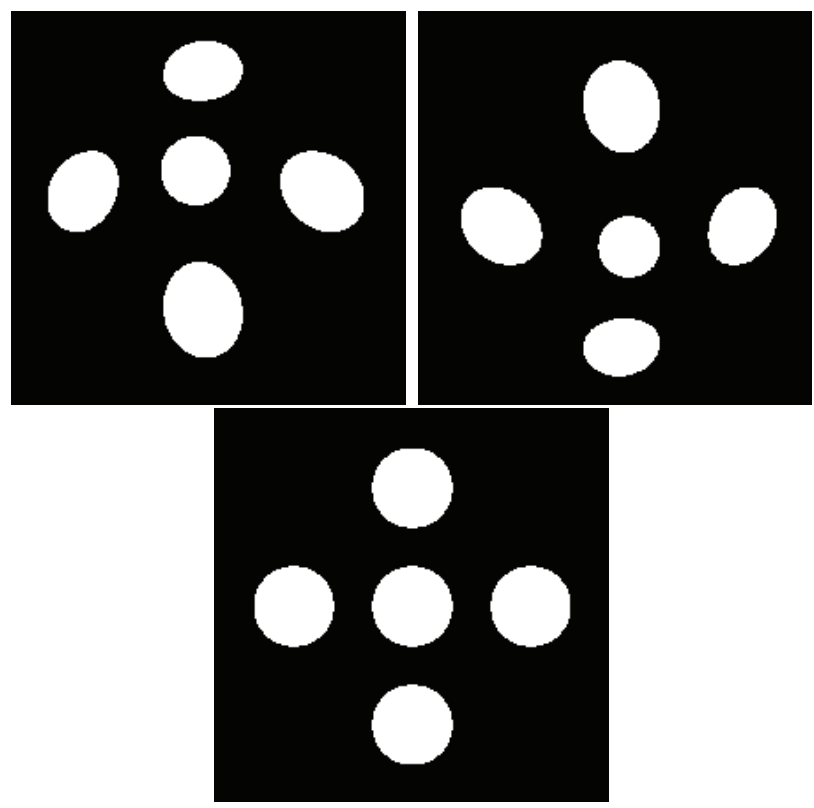

Figure 1. $x_{1} x_{2}$-cross-sections of the phantom at different times. Top left: at time corresponding to view 101. Top right: at time corresponding to view 900. Bottom: at reference time (view 501).

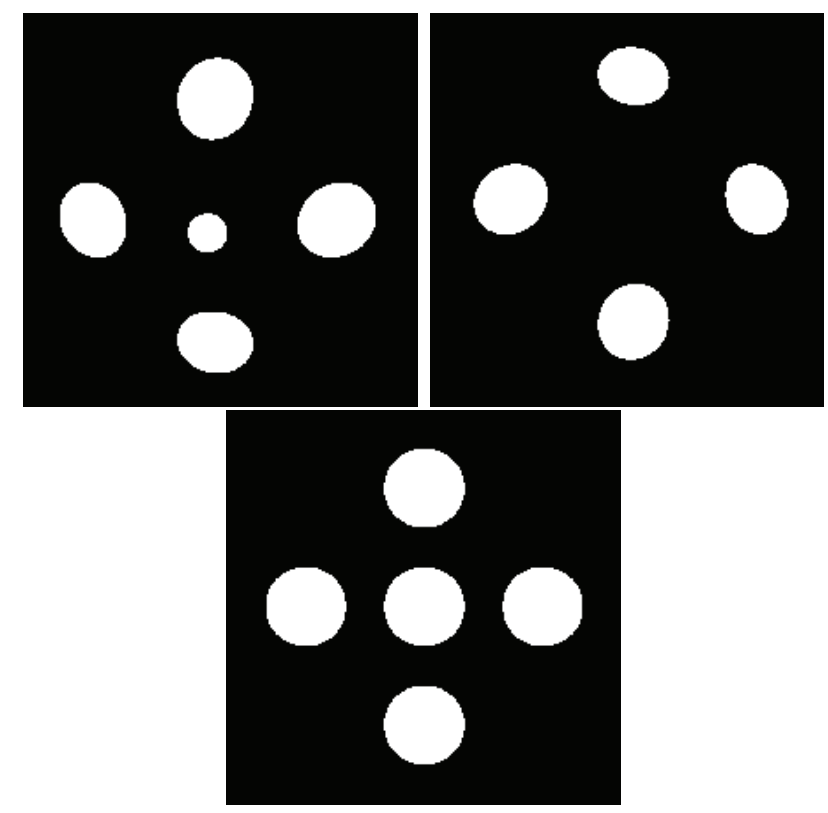

Figure 2. $x_{1} x_{3}$-cross-sections of the phantom at different times. Top left: at time corresponding to view 101. Top right: at time corresponding to view 900. Bottom: at reference time (view 501). 


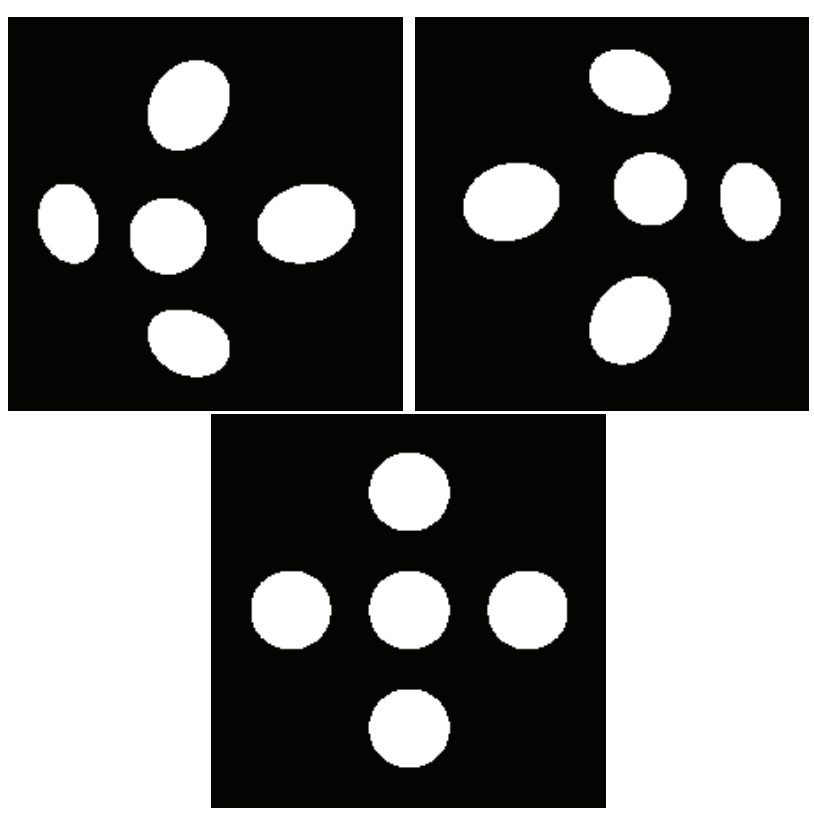

Figure 3. $x_{2} x_{3}$-cross-sections of the phantom at different times. Top left: at time corresponding to view 101. Top right: at time corresponding to view 900. Bottom: at reference time (view 501).

of the six planes that form its boundary. Minimization of edge entropy uses this observation and is done using the following approach.

Step 1. Let $a_{i k}, i=1, \ldots N_{k}, k=1,2,3$, be the current best estimate of the motion parameters. Let some $\Delta a \neq 0$ be given. Pick one of the $D_{l}$ 's. Let $a_{i_{1} k_{1}}, \ldots, a_{i_{6} k_{6}}$ be the six parameters affecting the chosen region. Compute $3^{6}$ subimages $\mathcal{B} f(x), x \in D_{l}$, corresponding to the sets $\tilde{a}_{i_{1} k_{1}}, \ldots, \tilde{a}_{i_{6} k_{6}}$, where each $\tilde{a}_{i k}$ equals either $a_{i k}$, or $a_{i k}-\Delta a$, or $a_{i k}+\Delta a$. Store all the subimages on the disk, and repeat for all $D_{l}$ 's.

Step 2. Run the loop over all $3^{N_{1}+N_{2}+N_{3}}$ sets $a_{i k}, i=1, \ldots N_{k}, k=1,2,3$, where each $\tilde{a}_{i k}$ equals either $a_{i k}$, or $a_{i k}-\Delta a$, or $a_{i k}+\Delta a$. This is done by reading the appropriate subimages from the disk and combining them into a single image of $\mathcal{B} f(x), x \in D$. Then compute edge entropy for the obtained image. From the $3^{N_{1}+N_{2}+N_{3}}$ sets of parameters find the one which produces the image with the smallest entropy.

Steps 1 and 2 constitute a single iteration. The initial values of $a_{i k}$ are chosen to be zero (which is the no motion assumption). The value of $\Delta a$ is chosen from some a priori considerations. After the end of each iteration, the optimal set of parameters identified at Step 2 is passed on to Step 1. Also, the value of $\Delta a$ is decreased. In our experiments we used $\Delta a_{\text {initial }}=10, \Delta a_{\text {new }}=0.75 \Delta a_{\text {old }}$, and three iterations were performed.

Results of experiments are shown in Figures 4 and 5. Figure 4 shows the initial image of $\mathcal{B} f$ computed under the (incorrect) assumption of no motion. Figure 5 shows the final image of $\mathcal{B} f$ computed for the motion model, which was determined by the algorithm. In these experiments we used $N_{1}=N_{2}=N_{3}=4$ (cf. (6.2)). We used $\kappa=0.0125$ to compute edge entropy. At the beginning of iterations (Figure 4) the value of entropy is $9.81 \%$, and at the end it is $8.40 \%$ (Figure 5). 


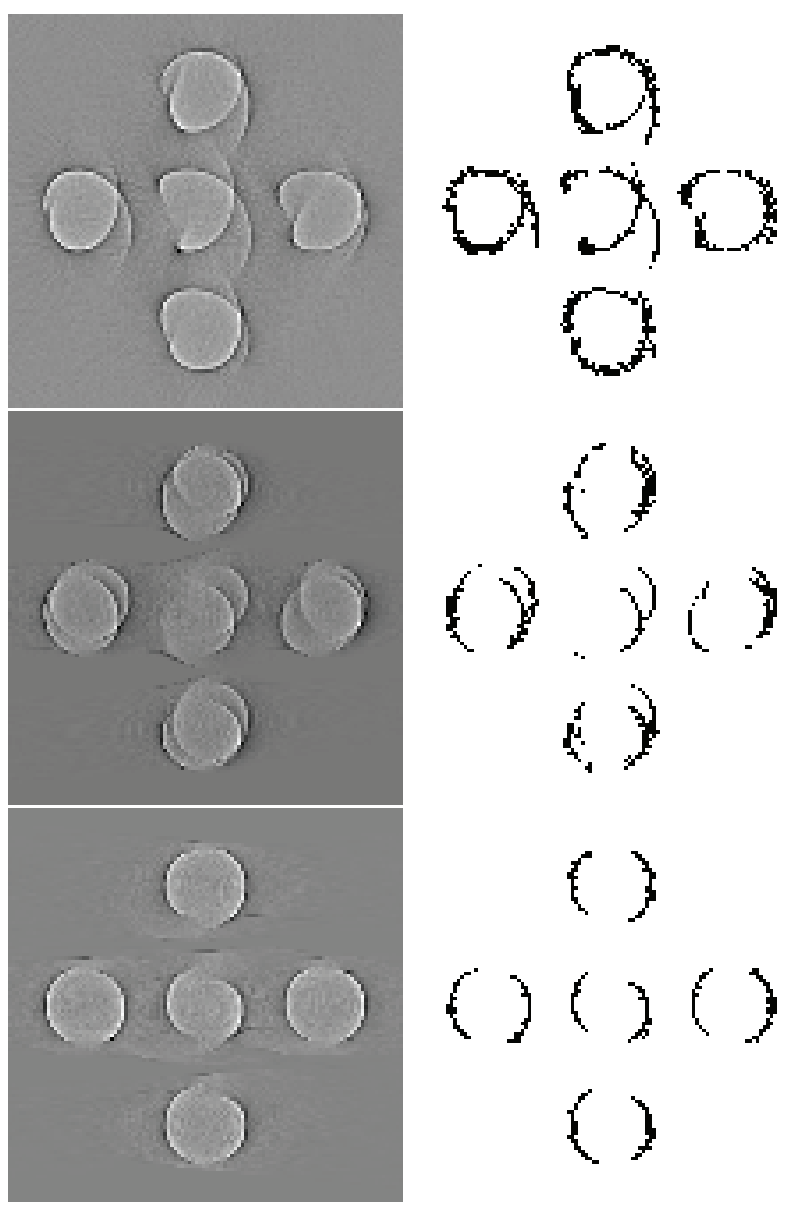

Figure 4. Left panel: density plots of $\mathcal{B} f$ at the beginning of iterations, i.e., when zero motion is assumed. Right panel: corresponding images of bright points. Top to bottom: cross-sections $x_{1} x_{2}, x_{1} x_{3}$, and $x_{2} x_{3}$ through the center of the grid. Entropy $9.81 \%$.

To illustrate another application of the proposed approach, we use it for solving a misalignment correction problem in the case of a distorted circular scan. Suppose that under the ideal circumstances the source trajectory is a circle. Suppose that because of mechanical instabilities the actual trajectory is a distorted circle given by

$$
x_{1}=R \cos s, \quad x_{2}=R \sin s, \quad x_{3}=\sum_{n=1}^{N} c_{n} \cos (n s) .
$$

Here $c_{n}, n=1,2, \ldots, N$, are unknown and are to be determined from the tomographic data. Suppose, for simplicity, that the detector always contains the $x_{3}$-axis, its center has the same $x_{3}$-coordinate as the source, and it is perpendicular to the source-to-center line. Thus, the detector moves along the $x_{3}$-axis in the same way as the source. It is clear that, similarly to the motion contaminated case, if the source trajectory is known with error, the edges spread and the image looks more random. Consequently, the procedure outlined at the end of 


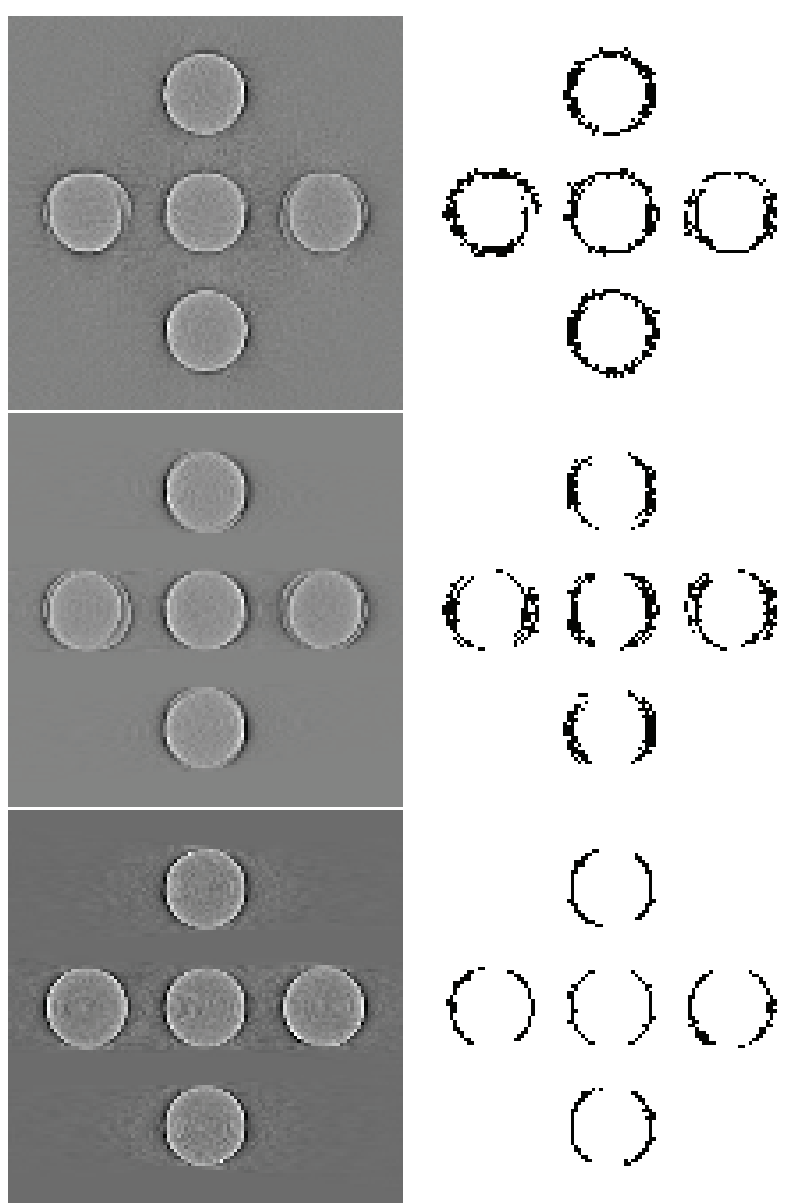

Figure 5. Left panel: density plots of $\mathcal{B} f$ at the end of iterations. Right panel: corresponding images of bright points. Top to bottom: cross-sections $x_{1} x_{2}, x_{1} x_{3}$, and $x_{2} x_{3}$ through the center of the grid. Entropy $8.40 \%$.

section 6 applies here as well (with the optimization of the "motion model" replaced by the optimization of the "trajectory model"). In our numerical experiment we used the same sevenball phantom as before (only it is not moving now) and took $N=5$ with $c_{1}=c_{3}=c_{5}=5$, $c_{2}=c_{4}=-5$. Other parameters, i.e., source-to-isocenter distance $R$ and size of reconstruction grid, were the same as in the first experiment. As initial approximation all $c_{i}$ 's were taken to be zero. Optimization was done using the Nelder-Mead simplex algorithm [23]. At the end of iterations the computed constants were $3.58,-5.87,4.81,-5.36,4.52$. Initial entropy was equal to $20.1 \%$, and at the end of iterations it was $8.44 \%$. See Figure 6 for the density plots of $\mathcal{B} f$.

In the case of noise-free (or low-noise) data, the algorithm based on LT as outlined above works well. If data are sufficiently noisy, the proposed scheme is unstable. Indeed, to compute the LT function we differentiate the data two times. Then, to find bright pixels, we differentiate the image one more time. To make the algorithm more robust, observe that instead of $\partial^{2} / \partial q^{2}$ in (2.5) we can use almost any even convolution kernel which preserves singular support. It 

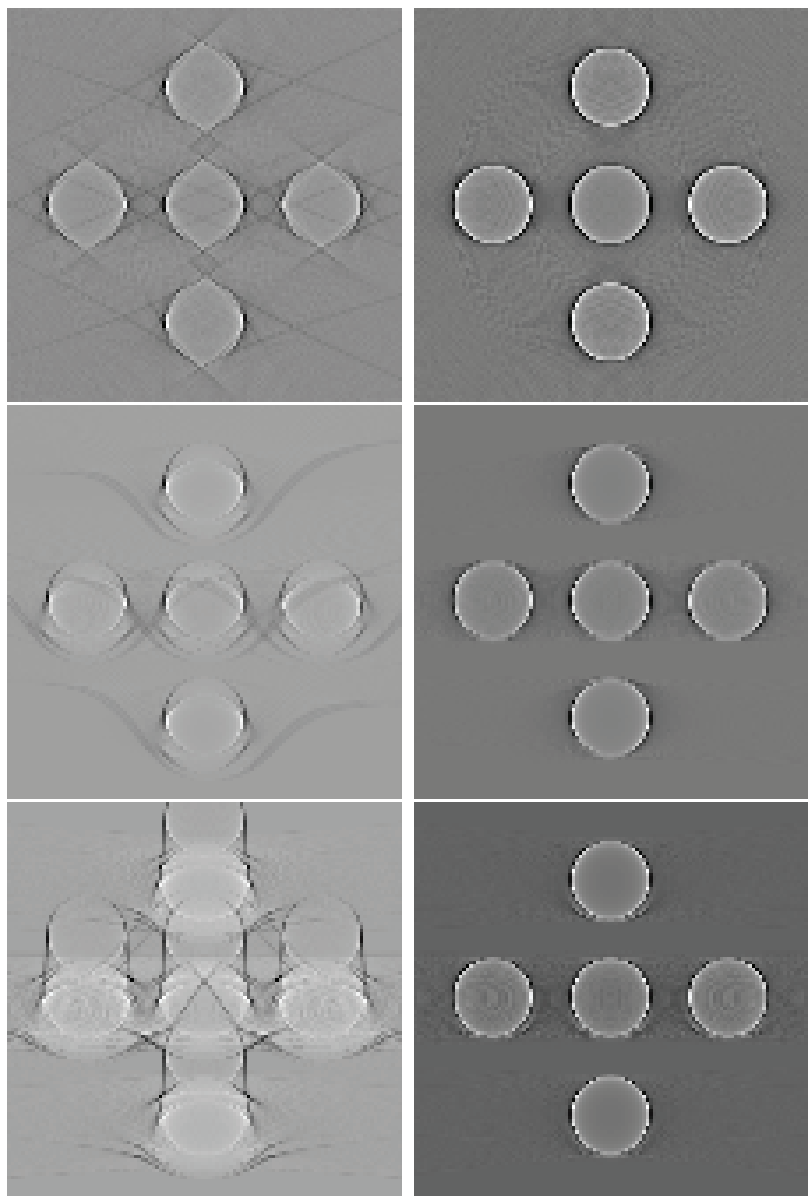

Figure 6. Left panel: density plots of $\mathcal{B} f$ at the beginning of iterations, i.e., when a pure circular trajectory is assumed (entropy 20.1\%). Right panel: density plots of $\mathcal{B} f$ at the end of iterations (entropy 8.44\%). Top to bottom: cross-sections $x_{1} x_{2}, x_{1} x_{3}$, and $x_{2} x_{3}$ through the center of the grid.

was found empirically that convolving the tomographic data along data rows with the kernel, whose frequency characteristic is given by $|\lambda|^{0.2}$, produces good results. In Figure 7 (left and middle panels), we see the LT images and the results of bright pixel detection using the filter $\partial^{2} / \partial q^{2}$ in (2.5) in the case of (erroneous assumption of) zero motion in the data. The right panel of Figure 7 shows the results of bright pixel detection using the new filter. As is seen, the latter is much less noisy than the former.

In Figure 8 we see the reconstructions at the end of iterative motion estimation. The left panel shows the intermediate "local" tomography image, and the middle panel shows the results of bright pixel detection. Note that these images are computed from the noisy data in the process of iterations and correspond to the motion model with the least edge entropy. Even though the filter is no longer local, we keep using the name "local tomography" to emphasize the fact that we still do not reconstruct density values (as opposed to conventional "global tomography"). To better illustrate our results we computed the traditional LT function from the noise-free data using the computed motion model; see the right panel. It is clearly seen

Copyright (c) by SIAM. Unauthorized reproduction of this article is prohibited. 

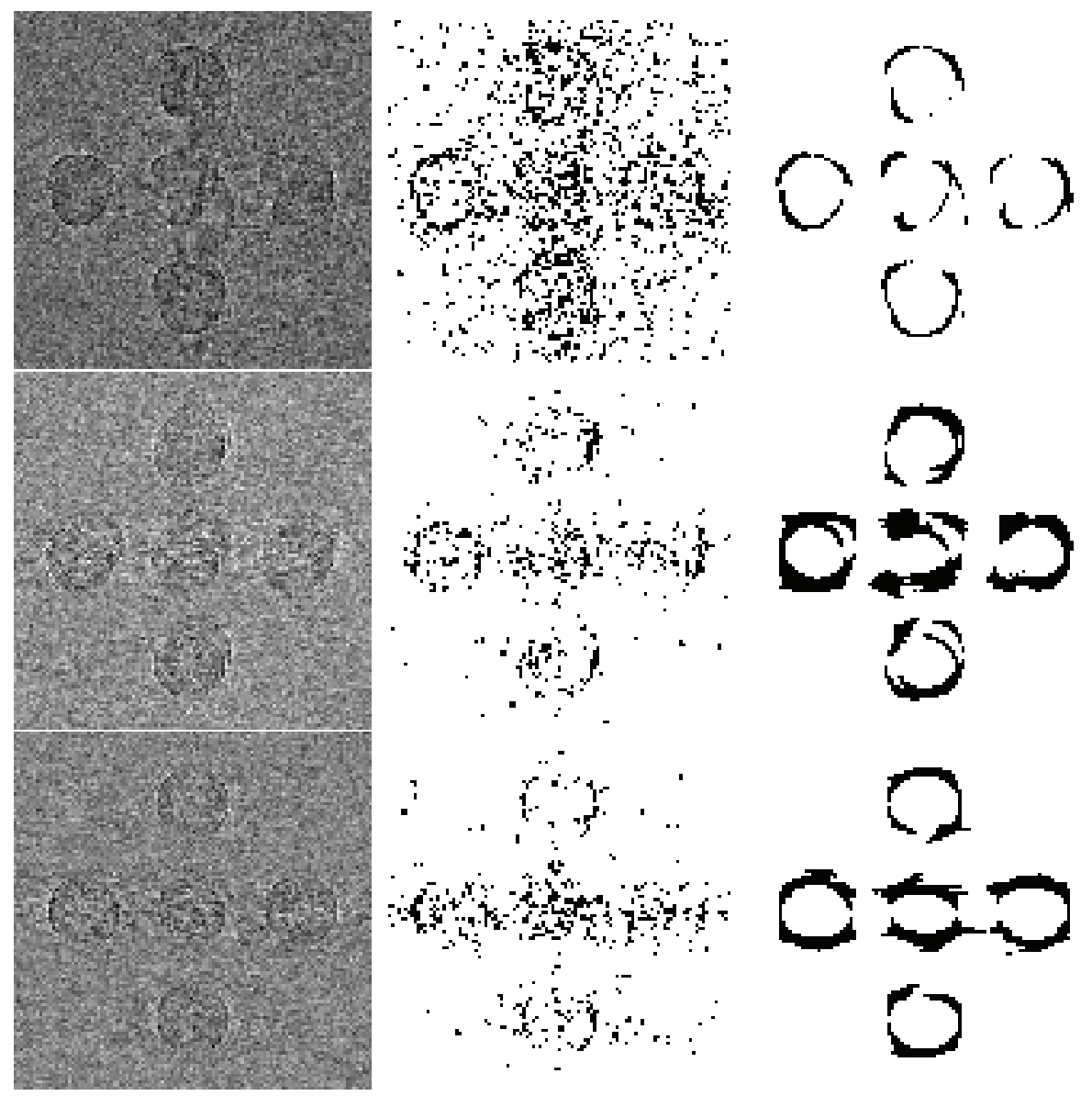

Figure 7. Left panel: density plots of $\mathcal{B} f$ at the beginning of iterations based on $\partial^{2} / \partial q^{2}$. Middle panel: the results of bright pixel detection using the LT function on the left. Right panel: the results of bright pixel detection using the LT function based on the new filter, entropy $8.82 \%$.

from these images that the edges are now much less spread out than they were at the beginning of iterations.

8. Discussion. In this paper we study LT in the motion contaminated case. It is shown that, microlocally, away from some critical directions, LT is equivalent to a pseudodifferential operator of order one. LT also produces nonlocal artifacts that are of the same strength as useful singularities. As opposed to the static case (cf. [17]), here it is not possible to choose the direction of differentiation to reduce the strength of the artifact by one order in the scale of Sobolev spaces. On the other hand, if motion is sufficiently small, it is expected that choosing $\Theta$ as in (2.31), which is analogous to what was done in [17] (compare (2.32) with (2.11) in [17]), may help reduce the artifacts. Finding the optimal direction of differentiation should be the subject of future work. Then we consider the case when motion is not accurately known. It is shown that when a singularity is seen from two different source positions, it spreads in different directions. A single edge becomes a double edge. Based on this observation we propose an algorithm for motion estimation. The algorithm is quite flexible and is used for 

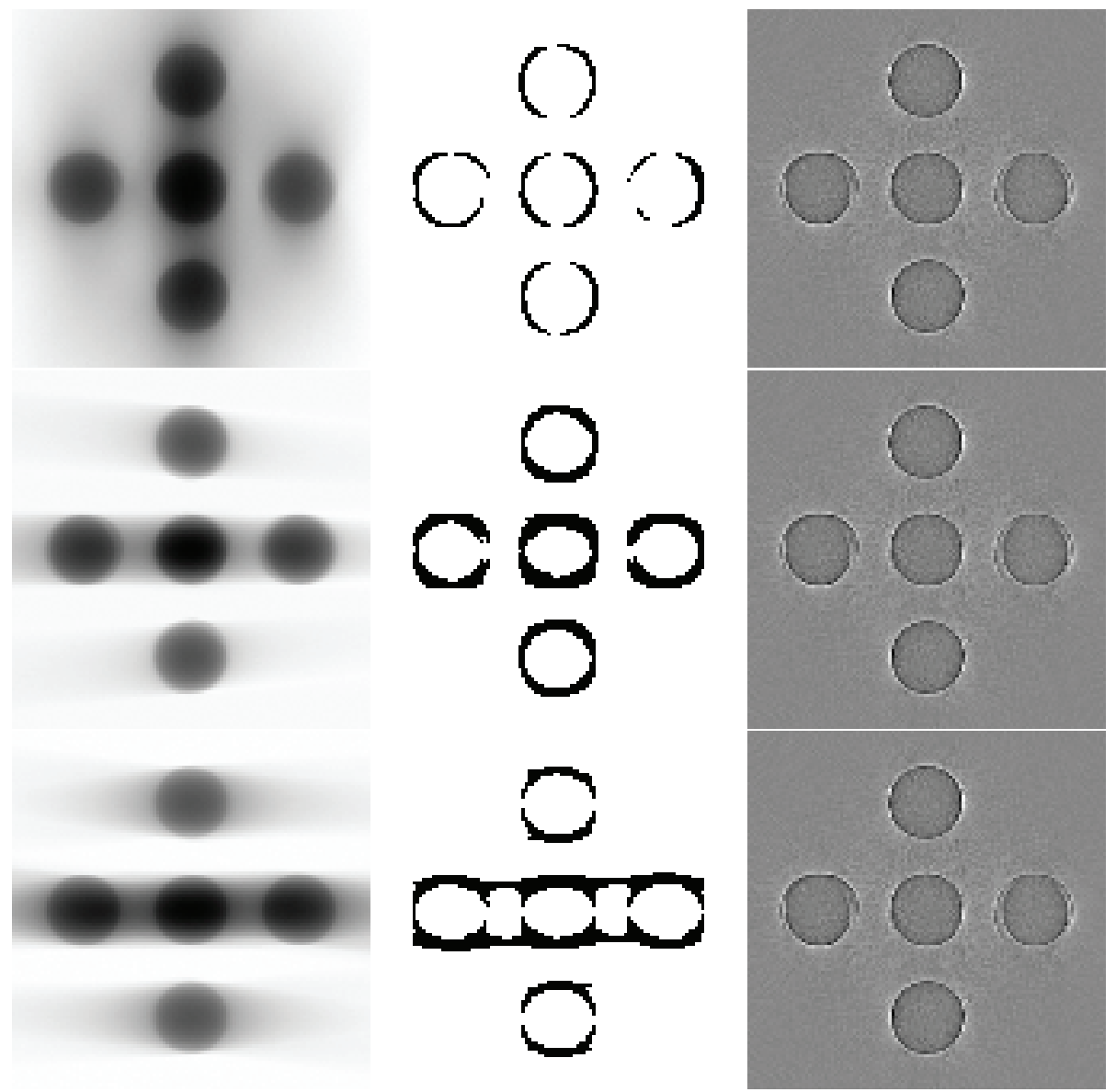

Figure 8. Left panel: density plots of $\mathcal{B} f$ at the end of iterations based on the new filter. Middle panel: the results of bright pixel detection using the LT function on the left, entropy $7.56 \%$. Right panel: classical motion-compensated LT function using the estimated motion model and noise-free data (for illustration purposes only).

solving the misalignment correction problem. The results of numerical experiments are very encouraging. More detailed testing of the algorithm with respect to noise in the data, strength of motion in the object, etc., as well as optimization of the computational efficiency will be the subject of future work. We also plan to study the role of the function $\varphi$ in (2.5). If the support of $\varphi(s, x)$ in $s$ is sufficiently short, the simple motion model, which is linear in $s$ (see section 6.1), approximates the real motion sufficiently well. On the other hand, there could be too few "double edges" to perform robust motion estimation. Increasing the support of $\varphi$ may lead to more "double edges," but the linear motion model may become less accurate. The optimal $\varphi$ should be determined on a case-by-case basis.

\section{REFERENCES}

[1] M. Anastasio, Y. Zou, X. Pan, And E. Sidky, Local cone-beam tomography image reconstruction on chords, J. Opt. Soc. Amer. A, 24 (2007), pp. 1569-1579.

Copyright ( $)$ by SIAM. Unauthorized reproduction of this article is prohibited. 
[2] C. Blondel, G. Malandain, R. Vaillant, and N. Ayache, Reconstruction of coronary arteries from a single rotational X-ray projection sequence, IEEE Trans. Med. Imaging, 25 (2006), pp. 653-663.

[3] C. Blondel, R. Vaillant, G. Malandain, and N. Ayache, 3D tomographic reconstruction of coronary arteries using a precomputed $4 D$ motion field, Phys. Med. Biol., 49 (2004), pp. 2197-2208.

[4] S. Bonnet, A. Koenig, S. Roux, P. Hugonnard, R. Guillemaud, and P. Grangeat, Dynamic X-ray computed tomography, Proc. IEEE, 91 (2003), pp. 1574-1587.

[5] C. Bontus, P. Koken, M. Grass, and T. Köhler, CEnPiT: Helical cardiac CT reconstruction, Med. Phys., 33 (2006), pp. 2792-2799.

[6] L. Desbat, S. Roux, And P. Grangeat, Compensation of some time dependent deformations in tomography, IEEE Trans. Med. Imaging, 26 (2007), pp. 261-269.

[7] Y. V. Egorov And B.-W. Schulze, Pseudo-differential Operators, Singularities, Applications, Birkhäuser, Basel, 1997.

[8] D. Finch, I.-R. Lan, and G. Uhlmann, Microlocal analysis of the x-ray transform with sources on a curve, in Inside Out: Inverse Problems and Applications, G. Uhlmann, ed., Cambridge University Press, Cambridge, UK, 2003, pp. 193-218.

[9] T. Flohr And B. Ohnesorge, Heart rate adaptive optimization of spatial and temporal resolution for electrocardiogram-gated multislice spiral CT of the heart, J. Comput. Assist. Tomogr., 25 (2001), pp. 907-923.

[10] P. Grangeat, A. Koenig, T. Rodet, and S. Bonnet, Theoretical framework for a dynamic cone-beam reconstruction algorithm based on a dynamic particle model, Phys. Med. Biol., 47 (2002), pp. 26112625.

[11] M. Grass, R. Manzke, T. Nielsen, P. Koken, R. Proksa, M. Natanzon, and G. Shechter, Helical cardiac cone beam reconstruction using retrospective ECG gating, Phys. Med. Biol., 48 (2003), pp. 3069-3084.

[12] A. Greenleaf and G. Uhlmann, Nonlocal inversion formulas for the X-ray transform, Duke Math. J., 58 (1989), pp. 205-240.

[13] L. Hörmander, The Analysis of Linear Partial Differential Operators. IV. Fourier Integral Operators, Springer-Verlag, Berlin, 1985.

[14] A. A. Isola, M. Grass, And W. J. Niessen, Fully automatic nonrigid registration-based local motion estimation for motion-corrected iterative cardiac CT reconstruction, Med. Phys., 37 (2010), pp. 10931109.

[15] M. Kachelriess, S. Ulzheimer, And W. A. Kalender, ECG-correlated imaging of the heart with subsecond multislice spiral CT, IEEE Trans. Med. Imaging, 19 (2000), pp. 888-901.

[16] A. Katsevich, Cone beam local tomography, SIAM J. Appl. Math., 59 (1999), pp. 2224-2246.

[17] A. Katsevich, Improved cone beam local tomography, Inverse Problems, 22 (2006), pp. 627-643.

[18] A. Katsevich, Motion compensated local tomography, Inverse Problems, 24 (2008), 045013.

[19] P. Koken and M. Grass, Aperture weighted cardiac reconstruction for cone-beam CT, Phys. Med. Biol., 51 (2006), pp. 3433-3448.

[20] Y. Kyriakou, R. M. Lapp, L. Hillebrand, D. Ertel, and W. Kalender, Simultaneous misalignment correction for approximate circular cone-beam computed tomography, Phys. Med. Biol., 53 (2008), pp. 6267-6289.

[21] G. Lauritsch, J. Boese, L. Wigström, R. Fahrig, And H. Kemeth, Towards cardiac C-arm computed tomography, IEEE Trans. Med. Imaging, 25 (2006), pp. 922-934.

[22] A. K. Louis And P. MAass, Contour reconstruction in 3-D X-ray CT, IEEE Trans. Med. Imaging, 12 (1993), pp. 764-769.

[23] J. Nelder and R. Mead, A simplex method for function minimization, Comput. J., 7 (1965), pp. 308313.

[24] J. D. PACK AND F. Noo, Dynamic computed tomography with known motion field, in Medical Imaging 2004: Image Processing, Proceedings of the SPIE, Vol. 5370, J. M. Fitzpatrick and M. Sonka, eds., 2004, pp. 2097-2104.

[25] E. T. QUinto, Electron microscope tomography, Conference talk at workshop on Mathematical Methods in Emerging Modalities of Medical Imaging, Banff International Research Station, Banff, Canada, 2009.

[26] E. T. Quinto And O. ÖKTEM, Local tomography in electron microscopy, SIAM J. Appl. Math., 68 (2008), pp. $1282-1303$.

Copyright (c) by SIAM. Unauthorized reproduction of this article is prohibited. 
[27] M. Reyes, G. Malandain, P. M. Koulibaly, M. A. Gonzalez-Ballester, and J. Darcourt, Model-based respiratory motion compensation for emission tomography image reconstruction, Phys. Med. Biol., 52 (2007), pp. 3579-3600.

[28] S. Rit, D. Sarrut, AND L. Desbat, Comparison of analytic and algebraic methods for motioncompensated cone-beam CT reconstruction of the thorax, IEEE Trans. Med. Imaging, 28 (2009), pp. $1513-1525$.

[29] A. Schweikard, H. Shiomi, And J. Adler, Respiration tracking in radiosurgery without fiducials, Int. J. Med. Robot., 1 (2005), pp. 19-27.

[30] D. Shen, H. Sundar, Z. Xue, Y. Fan, And H. Litt, Consistent estimation of cardiac motions by $4 D$ image registration, in Medical Image Computing and Computer-Assisted Intervention, Lecture Notes in Comput. Sci. 3750, Springer-Verlag, Berlin, Heidelberg, 2005, pp. 902-910.

[31] K. TAGUChi AND H. Kudo, Motion compensated fan-beam reconstruction for computed tomography using derivative backprojection filtering approach, in Proceedings of the IXth International Conference on Fully 3D Reconstruction in Radiology and Nuclear Medicine, Lindau, Germany, 2007, pp. 433-436.

[32] U. van StevendaAl, C. Lorenz, J. von Berg, and M. Grass, Motion-compensated reconstruction in helical cardiac $C T$, in Proceedings of the IXth International Conference on Fully 3D Reconstruction in Radiology and Nuclear Medicine, Lindau, Germany, 2007, pp. 437-440.

[33] M. Wierzbicki, G. M. Guiraudon, D. L. Jones, and T. Peters, Dose reduction for cardiac CT using a registration-based approach, Med. Phys., 34 (2007), pp. 1884-1895.

[34] Y. Ye, H. Yu, And G. WAng, Cone-beam pseudo-lambda tomography, Inverse Problems, 23 (2007), pp. 203-215.

[35] R. Zeng, J. A. Fessler, And J. M. Balter, Respiratory motion estimation from slowly rotating X-ray projections: Theory and simulation, Med. Phys., 32 (2005), pp. 984-991.

[36] R. Zeng, J. A. Fessler, And J. M. Balter, Estimating 3-D respiratory motion from orbiting views by tomographic image registration, IEEE Trans. Med. Imaging, 26 (2007), pp. 153-163.

Copyright $@$ by SIAM. Unauthorized reproduction of this article is prohibited. 